\title{
Многомерные цепные дроби и оценки линейных форм
}

\author{
А. Н. Коробов (Москва)
}

Обобщения непрерывных дробей рассматривали Эйлер, Якоби, Перрон, Минковский, Вороной и ряд других математиков. Первый алгоритм обобщающий алгоритм непрерывных дробей на двумерный случай предложил Эйлер. Якоби видоизменил алгоритм Эйлера, придав вычислениям больше однообразия, и обнаружил его периодичность для некоторых кубических иррациональностей. Якоби также убедился на частных примерах, что алгоритм позволяет находить хорошие совместные рациональные приближения с одним знаменателем для двух действительных чисел.

П. Бахман установил необходимые и достаточные условия периодичности алгоритма Якоби, связанные с характером совместных приближений.

Вороной предложил свой вариант алгоритма Якоби и доказал его периодичность для кубических иррациональностей.

В 1907 году О. Перрон [13] в своей магистерской диссертации обобщил алгоритм Якоби на многомерный случай и доказал ряд его основных свойств: сходимость, однозначность, линейную независимость получающихся чисел.

В последнее время появилось много исследований, посвященных алгоритму Якоби-Перрона и другим обобщениям непрерывных дробей. Так в работе Л. Бернштейна [10] исследовались вопросы, связанные с периодичностью алгоритма Якоби-Перрона. В диссертации А. Брентьеса [11] дается обзор большинства известных обобщений алгоритмов непрерывных дробей и исследуются свойства совместных приближений, обеспечиваемых алгоритмами.

Однако до настоящего времени по-видимому не было известно примеров разложений в многомерную цепную дробь векторов с трансцендентными компонентами и с помощью многомерных цепных дробей не получались точные оценки линейных форм от трансцендентных чисел. 
Заметим, что ранее получение нетривиальных точных оценок линейных форм было основано на построении функциональных аппроксимаций (аппроксимаций Паде [5]). Таким путем в работе А. И. Галочкина [2] были впервые получены оценки по порядку такие же, как и для почти всех чисел. В работе автора [3] были получены неулучшаемые оценки линейных форм с помощью применения линейного рекуррентного соотношения. По существу в этой работе было построено многомерное обобщение цепной дроби Гурвица. В работе А. И. Галочкина [1] методом функциональных аппроксимаций были затем получены более общие неулучшаемые оценки.

В настоящей статье приведены примеры разложения в многомерные цепные дроби систем чисел, обобщающие классические цепные дроби Эйлера, Гурвица и Рамануджана. В качестве следствия получены неулучшаемые оценки линейных форм от соответствующих чисел.

Получение неулучшаемых оценок основано на общей теореме о цепных дробях Якоби-Перрона специального вида. Метод доказательства этой теоремы следует работе автора [3]. В частности получены неулучшаемые оценки линейной формы от $\psi(1 / q), \psi^{\prime}(1 / q), \ldots$ $\ldots, \psi^{(s)}(1 / q)$, где $q \in \mathbb{N}$ и

$$
\psi(z)=\sum_{n=0}^{\infty} \frac{z^{n}}{((s+1) n) !}, \quad s \in \mathbb{N} .
$$

1. Основные свойства многомерных цепных дробей. В этой статье будут рассматриваться выражения вида

$$
\begin{gathered}
\vec{a}_{0}+\frac{1}{\vec{a}_{1}+\frac{1}{\ldots}}, \\
\vec{a}_{n}=\left(a_{1}(n), \ldots, a_{s}(n)\right) \in \mathbb{R}^{s}, \\
a_{i}(n) \geq 0, \quad a_{s}(n)>0, \quad i=1, \ldots, s, n=1,2, \ldots, s \in \mathbb{N},
\end{gathered}
$$

которые назовем $s$-мерными цепными дробями (или цепными $s$-дробями) с элементами $\vec{a}_{n}$.

Для того, чтобы приписать конечной цепной $s$-дроби

$$
\vec{\alpha}=\vec{a}_{0}+\frac{1}{\vec{a}_{1}+\frac{1}{\ldots+\frac{1}{\vec{a}_{n}}}}=\left[\vec{a}_{0} ; \vec{a}_{1}, \ldots, \vec{a}_{n}\right]
$$


определенное значение, положим

$$
1 /\left(\alpha_{1}, \ldots, \alpha_{s}\right)=\left(1 / \alpha_{s}, \alpha_{1} / \alpha_{s}, \ldots, \alpha_{s-1} / \alpha_{s}\right) .
$$

Теперь, согласно (3), каждой конечной $s$-дроби (2) соответствует вполне определенный $s$-мерный вектор (значение конечной $s$-дроби), причем при вычислении значения $s$-дроби не может возникнуть ситуация деления на ноль, поскольку

$$
a_{s}(k)>0, \quad a_{i}(k) \geq 0 \quad(k=1,2, \ldots, i=1, \ldots, s) .
$$

Цепные $s$-дроби (1) будем записывать также в виде $\left[\vec{a}_{0} ; \vec{a}_{1}, \vec{a}_{2}, \ldots\right]$.

Подходящей $s$-дробъю бесконечной цепной $s$-дроби (1) назовем вектор

$$
\vec{r}_{k}=\left[\vec{a}_{0} ; \vec{a}_{1}, \vec{a}_{2}, \ldots, \vec{a}_{k}\right], \quad k=0,1, \ldots
$$

Предположим, что существует предел $\vec{\alpha}=\lim _{k \rightarrow \infty} \vec{r}_{k}$. В этом случае $s$-дробь (1) назовем сходящейся к значению $\vec{\alpha}$ и будем писать $\vec{\alpha}=\left[\vec{a}_{0} ; \vec{a}_{1}, \vec{a}_{2}, \ldots\right]$.

Для любой сходящейся $s$-дроби (1) справедливо соотношение

$$
\vec{\alpha}=\left[\vec{a}_{0} ; \vec{a}_{1}, \ldots, \vec{a}_{k-1}, \vec{\alpha}_{k}\right]
$$

где $\vec{\alpha}_{k}=\left[\vec{a}_{k} ; \vec{a}_{k+1}, \vec{a}_{k+2}, \ldots\right]$. Из (4) следует равенство $\vec{\alpha}_{k}=\vec{a}_{k}+$ $1 / \vec{\alpha}_{k+1}, k=0,1, \ldots$, или, в координатной записи,

$$
\begin{aligned}
& \left(\alpha_{1}(k), \ldots, \alpha_{s}(k)\right)=\left(a_{1}(k), \ldots, a_{s}(k)\right) \\
& \quad+\left(1 / \alpha_{s}(k+1), \alpha_{1}(k+1) / \alpha_{s}(k+1), \ldots, \alpha_{s-1}(k+1) / \alpha_{s}(k+1)\right) .
\end{aligned}
$$

Запишем соотношение (5) в матричной форме

$$
\left[\begin{array}{c}
1 \\
\alpha_{1}(k) \\
\vdots \\
\alpha_{s}(k)
\end{array}\right]=\frac{1}{\alpha_{s}(k+1)} \cdot\left[\begin{array}{cccc}
0 & \ldots & 0 & 1 \\
1 & \ldots & 0 & a_{1}(k) \\
\ldots \ldots & \ldots . . . \\
0 & \ldots & 1 & a_{s}(k)
\end{array}\right] \cdot\left[\begin{array}{c}
1 \\
\alpha_{1}(k+1) \\
\vdots \\
\alpha_{s}(k+1)
\end{array}\right] .
$$

Из этого равенства получим

(6)

$$
\left[\begin{array}{c}
1 \\
\alpha_{1} \\
\vdots \\
\alpha_{s}
\end{array}\right]=\frac{1}{\alpha_{s}(1) \ldots \alpha_{s}(n+1)} \prod_{k=0}^{n}\left[\begin{array}{cccc}
0 & \ldots & 0 & 1 \\
1 & \ldots & 0 & a_{1}(k) \\
\ldots \ldots & \ldots & \ldots \\
0 & \ldots & 1 & a_{s}(k)
\end{array}\right] \cdot\left[\begin{array}{c}
1 \\
\alpha_{1}(n+1) \\
\vdots \\
\alpha_{s}(n+1)
\end{array}\right]
$$

Определим величины $A_{i}(n)$ из рекуррентных соотношений

$$
A_{i}(n+s+1)=A_{i}(n)+a_{1}(n) A_{i}(n+1)+\ldots+a_{s}(n) A_{i}(n+s)
$$

и начальных условий

$$
A_{i}(j)=\delta_{i j}, \quad i, j=0, \ldots, s .
$$


Индукцией легко установить, что тогда

$$
\prod_{k=0}^{n-1}\left[\begin{array}{cccc}
0 & \ldots & 0 & 1 \\
1 & \ldots & 0 & a_{1}(k) \\
\ldots & \ldots & \ldots & \ldots \\
0 & \ldots & 1 & a_{s}(k)
\end{array}\right]=\left[\begin{array}{ccc}
A_{0}(n) & \ldots & A_{0}(n+s) \\
A_{1}(n) & \ldots & A_{1}(n+s) \\
\ldots \ldots \ldots \ldots . \ldots \ldots \ldots \\
A_{s}(n) & \ldots & A_{s}(n+s)
\end{array}\right] .
$$

Из (6) теперь получаем равенства

$$
\begin{gathered}
\alpha_{i}=\frac{A_{i}(n)+A_{i}(n+1) \alpha_{1}(n)+\ldots+A_{i}(n+s) \alpha_{s}(n)}{A_{0}(n)+A_{0}(n+1) \alpha_{1}(n)+\ldots+A_{0}(n+s) \alpha_{s}(n)}, \\
\text { (9) } \alpha_{s}(1) \ldots \alpha_{s}(n)=A_{0}(n)+A_{0}(n+1) \alpha_{1}(n)+\ldots+A_{0}(n+s) \alpha_{s}(n), \\
\text { (10) }\left[\vec{a}_{0} ; \vec{a}_{1}, \ldots, \vec{a}_{n}\right]=\left(\frac{A_{1}(n+s+1)}{A_{0}(n+s+1)}, \ldots, \frac{A_{s}(n+s+1)}{A_{0}(n+s+1)}\right) .
\end{gathered}
$$

Заметим, что из равенств (5)-(10) следует равносильность понятия многомерной цепной дроби и алгоритма рассматривавшегося в работе Перрона [13].

Приведем критерий сходимости $s$-мерных цепных дробей, принадлежащий О. Перрону [13].

КРИТЕРИЙ ПЕРРона. Если

$$
\lim _{n \rightarrow \infty} \sup a_{i}(n) / a_{s}(n)<\infty \quad\left(i=0, \ldots, s-1, a_{0}(n)=1\right),
$$

то чепная $s$-дробь $\left[\vec{a}_{0} ; \vec{a}_{1}, \vec{a}_{2}, \ldots\right]$ сходится.

\section{2. Алгоритм Якоби-Перрона и многомерные цепные дроби.}

Пусть задан некоторый $s$-мерный вектор $\vec{\alpha}=\left(\alpha_{1}, \ldots, \alpha_{s}\right) \in \mathbb{R}^{s}$ такой, что $1, \alpha_{1}, \ldots, \alpha_{s}$ линейно независимы над $\mathbb{Q}$.

Алгоритм Якоби-Перрона состоит в нахождении векторов неполных частных $\vec{a}_{n}=\left(a_{1}(n), \ldots, a_{s}(n)\right)$ и векторов-остатков $\vec{\alpha}_{n}=\left(\alpha_{1}(n)\right.$, $\left.\ldots, \alpha_{s}(n)\right)$ по рекуррентным формулам

$$
\begin{aligned}
\vec{\alpha} & =\vec{\alpha}_{0}=\left(\alpha_{1}(0), \ldots, \alpha_{s}(0)\right), \\
\vec{a}_{n} & =\left(a_{1}(n), \ldots, a_{s}(n)\right)=\left(\left[\alpha_{1}(n)\right], \ldots,\left[\alpha_{s}(n)\right]\right), \\
\vec{\alpha}_{n} & =\vec{a}_{n}+1 / \vec{\alpha}_{n+1} .
\end{aligned}
$$

Пусть $\beta_{1} \neq 0$ и

$$
\left(\beta_{1}, \ldots, \beta_{s}\right)=1 /\left(\alpha_{1}, \ldots, \alpha_{s}\right)=\left(1 / \alpha_{s}, \alpha_{1} / \alpha_{s}, \ldots, \alpha_{s-1} / \alpha_{s}\right) .
$$

Тогда вектор $\left(\alpha_{1}, \ldots, \alpha_{s}\right)$ однозначно определяется по формуле

$$
\left(\alpha_{1}, \ldots, \alpha_{s}\right)=\left(\beta_{2} / \beta_{1}, \ldots, \beta_{s} / \beta_{1}, 1 / \beta_{1}\right) .
$$

Следовательно, последнее из равенств (11) можно переписать в 
виде

(12) $\quad \vec{\alpha}_{n+1}=\left(\frac{\alpha_{2}(n)-a_{2}(n)}{\alpha_{1}(n)-a_{1}(n)}, \ldots, \frac{\alpha_{s}(n)-a_{s}(n)}{\alpha_{1}(n)-a_{1}(n)}, \frac{1}{\alpha_{1}(n)-a_{1}(n)}\right)$.

Если $1, \alpha_{1}(n), \ldots, \alpha_{s}(n)$ линейно независимы над $\mathbb{Q}$, то $\alpha_{1}(n)-$ $a_{1}(n) \neq 0$ и в силу $(12), 1, \alpha_{1}(n+1), \ldots, \alpha_{s}(n+1)$ линейно независимы над $\mathbb{Q}$. Так как $1, \alpha_{1}(n), \ldots, \alpha_{s}(n)$ линейно независимы над $\mathbb{Q}$ при $n=0$, то это справедливо при любом $n \in \mathbb{N}$ и поэтому при любом $n=0,1, \ldots$ применима формула (12).

Таким образом, рекуррентные формулы (11) и (12) позволяют получить бесконечную последовательность векторов $\vec{a}_{n} \in \mathbb{Z}^{s}$ и $\vec{\alpha}_{n} \in$ $\mathbb{R}^{s}$ таких, что при $n=0,1, \ldots$ справедливы равенства

(13) $\vec{\alpha}=\vec{a}_{0}+1 / \vec{\alpha}_{1}=\vec{a}_{0}+1 /\left(\vec{a}_{1}+1 / \vec{\alpha}_{2}\right)=\ldots=\left[\vec{a}_{0} ; \vec{a}_{1}, \ldots, \vec{a}_{n}, \vec{\alpha}_{n+1}\right]$.

Кроме того, из (12) следует, что при $n=0,1, \ldots$ выполняются оценки

$$
\alpha_{s}(n+1)>\alpha_{i}(n+1)>0, \quad i=0, \ldots, s-1 \quad\left(\alpha_{0}(n)=1\right)
$$

и поскольку $a_{i}(n)=\left[\alpha_{i}(n)\right], i=1, \ldots, s$, то

$$
a_{i}(n) \in \mathbb{Z}, \quad a_{s}(n+1) \geq a_{i}(n+1) \geq 0, \quad i=0, \ldots, s \quad\left(a_{0}(n)=1\right) .
$$

Согласно критерию Перрона условия (14) обеспечивают сходимость цепной $s$-дроби $\left[\vec{a}_{0} ; \vec{a}_{1}, \vec{a}_{2}, \ldots\right]$.

ЛЕмма 1. Если при $n=0,1,2, \ldots$ справедливо представление вектора $\vec{\alpha} \in \mathbb{R}^{s}$ в виде конечной иепной s-дроби $\vec{\alpha}=\left[\vec{a}_{0} ; \vec{a}_{1}, \vec{a}_{2}, \ldots, \vec{a}_{n}, \vec{\alpha}_{n+1}\right]$,

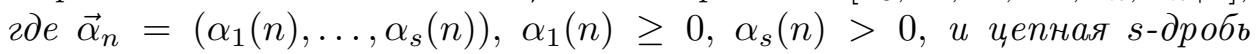
$\left[\vec{a}_{0} ; \vec{a}_{1}, \vec{a}_{2}, \ldots\right]$ сходится, то она сходится $\kappa$ вектору $\vec{\alpha}$ :

$$
\vec{\alpha}=\left[\vec{a}_{0} ; \vec{a}_{1}, \vec{a}_{2}, \ldots\right] \text {. }
$$

Доказательство. Заметим, что справедливо равенство (8), так как оно было выведено из соотношения (4), верного в условиях леммы.

Применяя (7) и (8), получаем

$$
\alpha_{i}=\sum_{j=0}^{s} \frac{A_{i}(n+j)}{A_{0}(n+j)} \lambda_{j}(n)
$$

где

$$
\begin{gathered}
\lambda_{j}(n)=\frac{A_{0}(n+j) a_{j}(n)}{\sum_{j=0}^{s} A_{0}(n+j) a_{j}(n)} \\
\sum_{j=0}^{s} \lambda_{j}(n)=1, \quad 0 \leq \lambda_{j}(n) \leq 1, \quad j=0, \ldots, s .
\end{gathered}
$$


Пусть $\left[\vec{a}_{0} ; \vec{a}_{1}, \ldots\right]=\vec{\beta}=\left(\beta_{1}, \ldots, \beta_{s}\right)$. Тогда, в силу $(10)$,

$$
\beta_{i}=\lim _{n \rightarrow \infty} A_{i}(n) / A_{0}(n), \quad i=1, \ldots, s,
$$

T.e.

$$
A_{i}(n) / A_{0}(n)=\beta_{i}+o(1) \quad(n \in \mathbb{N}) .
$$

Из (15) теперь следует, что

$$
\alpha_{i}=\sum_{j=0}^{s}\left(\beta_{i}+o(1)\right) \lambda_{j}(n)=\beta_{i}+o(1),
$$

т.е. $\alpha_{i}=\beta_{i}$ и лемма доказана.

Из леммы 1 и (13) следует, что алгоритм Якоби-Перрона позволяет разложить любой вектор $\vec{\alpha}=\left(\alpha_{1}, \ldots, \alpha_{s}\right)$ (такой, что $1, \alpha_{1}, \ldots$ $\ldots, \alpha_{s}$ линейно независимы над $\mathbb{Q}$ ) в сходящуюся цепную дробь:

$$
\vec{\alpha}=\left[\vec{a}_{0} ; \vec{a}_{1}, \vec{a}_{2}, \ldots\right], \quad \vec{a}_{n}=\left(a_{1}(n), \ldots, a_{s}(n)\right),
$$

причем выполнены условия (14).

3. Правильные многомерные цепные дроби и конечноразностные уравнения. Назовем цепную $s$-дробь (1) правильной, если ее элементы имеют вид

$$
\vec{a}_{n}=\left(0, \ldots, 0, a_{n}\right), \quad a_{n} \in \mathbb{N}, n=0,1, \ldots
$$

Понятие правильной $s$-дроби является обобщением понятия обычной цепной дроби с натуральными элементами (см., например, $[7])$. Для правильных $s$-дробей удается получить наиболее точные оценки соответствующих линейных форм и построить примеры разложения в такие дроби.

Из критерия Перрона следует, что любая правильная $s$-дробь сходится. Из результатов работы Перрона [13] также следует, что если разложение в правильную многомерную дробь существует, то оно единственно.

В следующей лемме устанавливается связь между положительными решениями некоторых рекуррентных уравнений и правильными $s$-дробями.

Лемма 2. Пусть $a_{n} \in \mathbb{N}(n=1,2, \ldots)$ и последовательность $x_{n}>0$ $(n=0,1, \ldots)$ положительных чисел удовлетворяет рекуррентному уравнению

$$
x_{n+s}=x_{n-1}-a_{n} x_{n} .
$$


Тогда справедливо разложение в правильную s-дробь

$$
\left(x_{1} / x_{0}, \ldots, x_{s} / x_{0}\right)=\frac{1}{\vec{a}_{1}+\frac{1}{\vec{a}_{2}+\ldots}},
$$

где $\vec{a}_{n}=\left(0, \ldots, 0, a_{n}\right)$.

Доказательство. Определим последовательность $s$-мерных векторов $\vec{\alpha}_{n}(n=1,2, \ldots)$ из равенства

$$
\vec{\alpha}_{n}=\left(\alpha_{1}(n), \ldots, \alpha_{s}(n)\right)=\left(x_{n+1} / x_{n}, \ldots, x_{n+s-1} / x_{n}, x_{n-1} / x_{n}\right) .
$$

Применяя (16), получаем

$$
\vec{\alpha}_{n}=\left(0, \ldots, 0, a_{n}\right)+\left(x_{n+1} / x_{n}, \ldots, x_{n+s} / x_{n}\right)=\vec{a}_{n}+1 / \vec{\alpha}_{n+1} .
$$

Следовательно, при $n=0,1,2, \ldots$ имеем

$$
\left(x_{1} / x_{0}, \ldots, x_{s} / x_{0}\right)=\left[0 ; \vec{\alpha}_{1}\right]=\left[0 ; \vec{a}_{1}, \vec{\alpha}_{2}\right]=\ldots=\left[0 ; \vec{a}_{1}, \ldots, \vec{a}_{n}, \vec{\alpha}_{n+1}\right] .
$$

Из леммы 1 теперь следует (17). Лемма доказана.

Покажем, что справедливо утверждение обратное лемме 2.

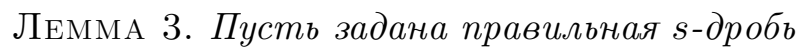

$$
\begin{gathered}
\left(\alpha_{1}, \ldots, \alpha_{s}\right)=\frac{1}{\vec{a}_{1}+\frac{1}{\vec{a}_{2}+\ldots}} \\
\vec{a}_{n}=\left(0, \ldots, 0, a_{n}\right), \quad\left(\alpha_{1}(n), \ldots, \alpha_{s}(n)\right)=\left[\vec{a}_{n} ; \vec{a}_{n+1}, \ldots\right] .
\end{gathered}
$$

Тогда последовательность

$$
x_{n}=1 /\left(\alpha_{s}(1) \ldots \alpha_{s}(n)\right), \quad n=0,1, \ldots \quad\left(x_{0}=1\right),
$$

удовлетворяет уравнению (16).

Доказательство. Для правильной $s$-дроби соотношения (5) примут вид

$$
\begin{aligned}
\alpha_{1}(n) & =\frac{1}{\alpha_{s}(n+1)}, \\
\alpha_{2}(n) & =\frac{\alpha_{1}(n+1)}{\alpha_{s}(n+1)}=\frac{1}{\alpha_{s}(n+1) \alpha_{s}(n+2)}, \ldots, \\
\alpha_{s-1}(n) & =\frac{\alpha_{s-2}(n+1)}{\alpha_{s}(n+1)}=\frac{1}{\alpha_{s}(n+1) \ldots \alpha_{s}(n+s-1)}, \\
\alpha_{s}(n) & =a_{n}+\frac{1}{\alpha_{s}(n+1) \ldots \alpha_{s}(n+s)} .
\end{aligned}
$$

Пользуясь (18), перепишем (19) следующим образом:

$$
\alpha_{s}(n)=x_{n-1} / x_{n}=a_{n}+x_{n+s} / x_{n},
$$

откуда $x_{n+s}=x_{n-1}-a_{n} x_{n}$. Лемма доказана. 
4. Общая теорема. Пусть некоторый $s$-мерный вектор разложен в правильную цепную $s$-дробь

$$
\begin{aligned}
& \left(\alpha_{1}, \ldots, \alpha_{s}\right)=\frac{1}{\vec{a}_{1}+\frac{1}{\vec{a}_{2}+\ldots},} \\
& \vec{a}_{n}=\left(0, \ldots, 0, a_{n}\right), \quad a_{n} \in \mathbb{N} .
\end{aligned}
$$

Согласно лемме 3 , последовательность $x_{n}=1 /\left(\alpha_{1}(1) \ldots \alpha_{s}(n)\right)$, где $\left(\alpha_{1}(n), \ldots, \alpha_{s}(n)\right)=\left[\vec{a}_{n} ; \vec{a}_{n+1}, \ldots\right]$, удовлетворяет уравнению $(16)$,

$$
x_{n+s}=x_{n-1}-a_{n} x_{n} .
$$

Определим целые числа $h_{n}(i)$ как решения этого уравнения с начальными условиями

$$
h_{j}(i)=\delta_{i j}, \quad j=0, \ldots, s .
$$

Лемма 4. Справедливы равенства

$$
\begin{gathered}
x_{n}=h_{n}(0) x_{0}+\ldots+h_{n}(s) x_{s}, \\
\left|\operatorname{det}\left(h_{n+j}(i)\right)\right|=1, \quad i, j=0, \ldots, s .
\end{gathered}
$$

Доказательство. Из (20) следует, что равенство (21) выполняется при $n=0, \ldots, s$, а равенство $(22)$ - при $n=0$. Предположим, что при некотором $k$ равенство (21) справедливо при $n=k-1, \ldots, k+s-1$ и (22) справедливо при $n=k-1$.

Пользуясь уравнением (16), получаем

$$
\begin{aligned}
x_{k+s} & =x_{k-1}-a_{k} x_{k} \\
& =\left(h_{k-1}(0)-a_{k} h_{k}(0)\right) x_{0}+\ldots+\left(h_{k-1}(s)-a_{k} h_{k}(s)\right) x_{s} \\
& =h_{k+s}(0) x_{0}+\ldots+h_{k+s}(s) x_{s} .
\end{aligned}
$$

Имеем

$$
\begin{aligned}
\mid \operatorname{det}\left(h_{k}(i), \ldots, h_{k+s}(i)\right) & \left.\right|_{(i=0, \ldots, s)} \\
& =\left|\operatorname{det}\left(h_{k}(i), \ldots, h_{k+s-i}(i), h_{k-1}(i)-a_{k} h_{k}(i)\right)\right| \\
& =\left|\operatorname{det}\left(h_{k}(i), \ldots, h_{k+s-1}(i), h_{k-1}(i)\right)\right|=1 .
\end{aligned}
$$

Таким образом, в силу индукции, лемма доказана.

Пусть задано разложение в правильную $s$-дробь

$$
\left(\alpha_{1}, \ldots, \alpha_{s}\right)=\frac{1}{\vec{a}_{1}+\frac{1}{\vec{a}_{2}+\ldots}}, \quad \vec{a}_{n}=\left(0, \ldots, 0, a_{n}\right), a_{n} \in \mathbb{N} .
$$

Определим величины $H_{n} \in \mathbb{N}$ следующим образом:

$$
\begin{aligned}
H_{n} & =a_{n-s} a_{n-2 s} \ldots a_{r} \quad(1 \leq r \leq s, n \equiv r(\bmod s), n>s), \\
H_{0} & =\ldots=H_{s}=1
\end{aligned}
$$


Лемма 5. Если выполнены условия

$$
H_{n+1} \gg H_{n}, \quad \sum_{n=1}^{\infty} H_{n-1} / H_{n+s}<\infty,
$$

то справедливы оченки $(n=0,1, \ldots)$

$$
\begin{gathered}
\left|h_{n}(i)\right| \ll H_{n}, \\
x_{n}<\frac{1}{H_{n+1} \ldots H_{n+s}} .
\end{gathered}
$$

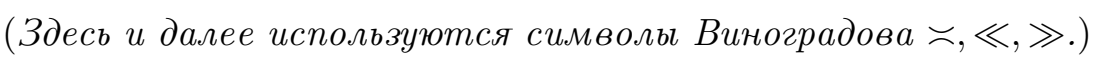

Доказательство. Положим $h_{n}(i)=u_{n} H_{n}$. Тогда уравнение (16) для $h_{n}(i)$ перепишется в виде

$$
\begin{gathered}
u_{n+s} H_{n+s}=u_{n-1} H_{n-1}-a_{n} u_{n} H_{n}=u_{n-1} H_{n-1}-u_{n} H_{n+s}, \\
u_{n+s}=-u_{n}+\left(H_{n-1} / H_{n+s}\right) u_{n-1} .
\end{gathered}
$$

Пусть $M_{n}=\max \left(\left|u_{n+s}\right|, \ldots,\left|u_{n}\right|\right)$. Тогда

$$
\begin{aligned}
M_{n} & \leq M_{n-1}\left(1+H_{n-1} / H_{n+s}\right) \leq \ldots \leq M_{0} \prod_{k=1}^{n}\left(1+H_{k-1} / H_{k+s}\right) \\
& \leq \prod_{k=1}^{\infty}\left(1+H_{k-1} / H_{k+s}\right) .
\end{aligned}
$$

Так как по условию $(24)$ ряд $\sum_{n=1}^{\infty} H_{n-1} / H_{n+s}$ сходится, то сходится и бесконечное произведение $\prod_{k=1}^{\infty}\left(1+H_{k-1} / H_{k+s}\right)$. Следовательно, $M_{n} \ll 1$ и получаем оценку $(25)$ :

$$
\left|h_{n}(i)\right|=\left|u_{n}\right| H_{n} \leq M_{n} H_{n} \ll H_{n} .
$$

Из (18) и (23) следует оценка (26):

$$
x_{n}=\frac{1}{\alpha_{s}(1) \ldots \alpha_{s}(n)}<\frac{1}{a_{1} \ldots a_{n}}=\frac{1}{H_{n+1} \ldots H_{n+s}} .
$$

Лемма доказана.

ТеОрема 1. Пусть для правильной чепной $s$-дроби

$$
\left(\alpha_{1}, \ldots, \alpha_{s}\right)=\frac{1}{\vec{a}_{1}+\frac{1}{\vec{a}_{2}+\ldots}}, \quad \vec{a}_{n}=\left(0, \ldots, 0, a_{n}\right), a_{n} \in \mathbb{N},
$$

выполнены условия (24) и для некоторой положительной и возрастающей функции $\Phi(x)$ выполняются оченки

$$
\begin{array}{r}
\Phi\left(H_{n}\right) \gg H_{n+1} \ldots H_{n+s}, \quad \Phi(x / 2) \gg \Phi(x) \\
\left(H_{n}=a_{n-s} a_{n-2 s} \ldots a_{r}(1 \leq r \leq s)\right) .
\end{array}
$$


Тогда для любих $h_{0}, \ldots, h_{s} \in \mathbb{Z}$ справедлива оченка

$$
\left|h_{0}+h_{1} \alpha_{1}+\ldots+h_{s} \alpha_{s}\right| \gg 1 / \Phi(H), \quad H=\max \left(\left|h_{0}\right|, \ldots,\left|h_{s}\right|\right)>0 .
$$

При этом существует последовательность ненулевых линейных форм с целыми коэффициентами таких, что

$$
\begin{gathered}
\left|h_{n}(0)+h_{n}(1) \alpha_{1}+\ldots+h_{n}(s) \alpha_{s}\right| \ll \frac{1}{H_{n+1} \ldots H_{n+s}}, \quad n=0,1, \ldots, \\
\left|h_{n}(i)\right| \ll H_{n}, \quad h_{n}(i) \in \mathbb{Z} \quad(i=0, \ldots, s) .
\end{gathered}
$$

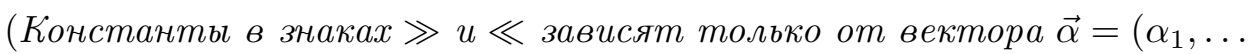
$\left.\left.\ldots, \alpha_{s}\right).\right)$

Доказательств в. Пусть $h_{0}, \ldots, h_{s} \in \mathbb{Z}$ и $H=\max \left(\left|h_{0}\right|, \ldots,\left|h_{s}\right|\right)$ $>0$. Так как, согласно $(22), \operatorname{det}\left(h_{n+j}(i)\right) \neq 0$, то при любом $n$ найдется такое $i$, что определитель

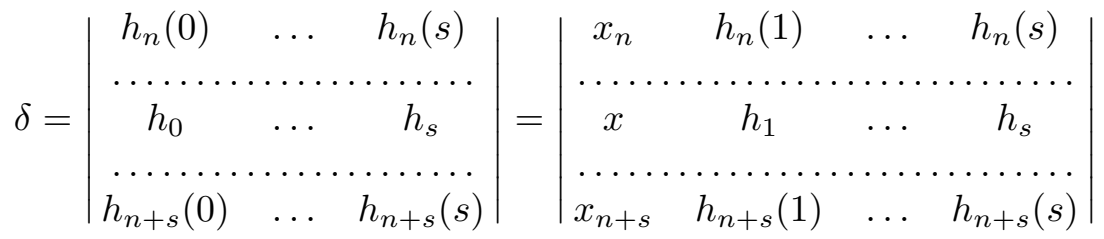

ненулевой, где

$$
x_{k}=h_{k}(0) x_{0}+\ldots+h_{k}(s) x_{s}, \quad x=h_{0} x_{0}+\ldots+h_{s} x_{s} \quad\left(x_{0}=1\right)
$$

(определитель $\delta$ получен заменой $i$-той строки определителя $\operatorname{det}\left(h_{n+j}(k)\right)$ на ненулевую строку $\left.\left(h_{0}, \ldots, h_{s}\right)\right)$. Так как $\delta \in \mathbb{Z}$ и $\delta \neq 0$, то $|\delta| \geq 1$, откуда следует, что

$$
\left|x A+\sum_{\substack{k=0 \\ k \neq i}}^{s} x_{n+k} A_{k}\right| \geq 1,
$$

где $A_{0}, \ldots, A_{i-1}, A, A_{i+1}, \ldots, A_{s}$ - алгебраические дополнения элементов первого столбца определителя $\delta$.

Применяя оценки леммы 5 и условия (24), получаем

$$
\begin{gathered}
|A| \ll \frac{H_{n} \ldots H_{n+s}}{H_{n+1}} \ll H_{n+1} \ldots H_{n+s}, \\
\left|A_{k}\right| \ll \frac{H H_{n} \ldots H_{n+s}}{H_{n+i} H_{n+k}}, \quad k \neq i, \\
\left|x_{n+k}\right| \ll \frac{1}{H_{n+1} \ldots H_{n+s}}, \quad k=0, \ldots, s .
\end{gathered}
$$


Следовательно,

$$
\left|\sum_{\substack{k=0 \\ k \neq i}}^{s} x_{n+k} A_{k}\right| \ll \sum_{k \neq i} \frac{H H_{n}}{H_{n+i} H_{n+k}} \ll \frac{H}{H_{n+1}},
$$

т.е. для некоторой константы $c>0$,

$$
\left|\sum_{k \neq i} x_{n+k} A_{k}\right|<c H / H_{n+1} .
$$

Из условий (24) следует, что последовательность $H_{n}$ неограничена. Выберем наименьшее натуральное $n$, при котором $2 c H<H_{n+1}$. Тогда $H \gg H_{n}$ и из (28)-(30) получим

$$
|x| \gg \frac{1}{2|A|} \gg \frac{1}{H_{n+1} \ldots H_{n+s}} \gg \frac{1}{\Phi\left(H_{n}\right)} \gg \frac{1}{\Phi(H)} .
$$

Остается заметить, что в силу лемм $2-4$,

$$
\left(\alpha_{1}, \ldots, \alpha_{s}\right)=\left(x_{1}, \ldots, x_{s}\right), \quad|x|=\left|h_{0}+h_{1} \alpha_{1}+\ldots+h_{s} \alpha_{s}\right| .
$$

Второе утверждение теоремы следует из леммы 5 . Теорема доказана.

5. Многомерные обобщения некоторых классических цепных дробей и точные по высоте оценки линейных форм. Будем называть оценку (27) теоремы 1 точной nо высоте, если существует бесконечная последовательность линейных форм вида

$$
L=h_{0}+h_{1} \alpha_{1}+\ldots+h_{s} \alpha_{s}, \quad h_{0}, \ldots, h_{s} \in \mathbb{Z},
$$

высоты $H=\max \left(\left|h_{0}\right|, \ldots,\left|h_{s}\right|\right)$, для которых $|L| \ll 1 / \Phi(H)$, т.е. уточнение оценки (27) возможно только за счет константы в знаке 》.

Ниже будут рассмотрены примеры правильных многомерных цепных дробей, обобщающие известные цепные дроби Гурвица, Эйлера-Ламберта и Рамануджана. В качестве примеров на теорему 1 будут получены точные по высоте оценки соответствующих линейных форм. Заметим, что подобные оценки рассматривались ранее в работах [3] и [2] вне связи с многомерными цепными дробями. Заметим также, что в одномерном случае $(s=1)$ связь цепных дробей и точных оценок мер иррациональности общеизвестна (см. $[4],[9])$.

1. Многомерная цепная дробь Гурвица. Рассмотрим последовательность $(n=0,1, \ldots)$ 


$$
\psi_{n}=\sum_{\nu=0}^{\infty} \frac{1}{(a s)^{\nu} \nu !} \prod_{x=1}^{n+s \nu}(a x+b)^{-1}, \quad a, a+b \in \mathbb{N} .
$$

Проверим, что выполняется равенство

$$
\psi_{n-1}-(a n+b) \psi_{n}=\psi_{n+s}, \quad n=1,2, \ldots
$$

Действительно,

$$
\begin{aligned}
\psi_{n-1}-(a n+b) \psi_{n} & =\sum_{\nu=0}^{\infty} \frac{a(n+s \nu)+b-(a n+b)}{(a s)^{\nu} \nu !} \prod_{x=1}^{n+s \nu}(a x+b)^{-1} \\
& =\sum_{\nu=1}^{\infty} \frac{1}{(a s)^{\nu-1}(\nu-1) !} \prod_{x=1}^{n+s \nu}(a x+b)^{-1}=\psi_{n+s} .
\end{aligned}
$$

Следовательно, на основании леммы 2 получаем разложение в правильную $s$-дробь

$$
\left(\psi_{1} / \psi_{0}, \ldots, \psi_{s} / \psi_{0}\right)=\left[0 ; \vec{a}_{1}, \vec{a}_{2}, \ldots\right], \quad \vec{a}_{n}=(0, \ldots, 0, a n+b) .
$$

В частности, при $s=1$ имеем известное разложение в цепную дробь Гурвица (см. [4])

$$
\psi_{1} / \psi_{0}=[0 ; a+b, 2 a+b, \ldots] .
$$

Для того, чтобы применить теорему 2, покажем, что выполняются оценки

$$
\begin{gathered}
\ln H_{n} \asymp n \ln n, \\
H_{n+1} \asymp n^{1 / s} H_{n} .
\end{gathered}
$$

Действительно,

$$
\begin{gathered}
H_{n}=(a(n-s)+b) \ldots(a r+b) \quad(1 \leq r \leq s), \\
\ln H_{n} \asymp \ln (n / s-1)+\ln (n / s-2)+\ldots+\ln (r / s)+O(n) \\
\asymp \ln ([n / s] !)+O(n) \asymp n \ln n, \\
\frac{H_{n+1}}{H_{n}} \asymp \prod_{k=1}^{[n / s]} \frac{a(n-k s+1)+b}{a(n-k s)+b}=\prod_{k=1}^{[n / s]}\left(1+\frac{1 / s}{n / s-k+b /(a s)}\right) .
\end{gathered}
$$

Логарифмируя, получаем

$$
\begin{aligned}
\ln \frac{H_{n+1}}{H_{n}} & =\sum_{k=1}^{[n / s]} \frac{1}{s} \cdot \frac{1}{n / s-k+b /(a s)}+O(1) \\
& =(1 / s) \ln (n / s)+O(1)=(1 / s) \ln n+O(1),
\end{aligned}
$$

т.е. $H_{n+1} / H_{n} \asymp n^{1 / s}$ и оценки (32) и (33) доказаны. 
Из (32) и (33) следует, что (24) выполнено и

$$
\begin{aligned}
H_{n+1} \ldots H_{n+s} & \asymp n^{1 / s+2 / s+\ldots+s / s} H_{n}^{s} \\
& =n^{(s+1) / 2} H_{n}^{s} \asymp H_{n}^{s}\left(\ln \left(H_{n}+2\right) / \ln \ln \left(H_{n}+2\right)\right)^{(s+1) / 2} .
\end{aligned}
$$

Теперь, применяя теорему 1 , в качестве примера на общую теорему получаем результат работы [3]:

Teоpema 2. Пyсmb $s, a, a+b \in \mathbb{N} u$

$$
\psi_{n}=\sum_{\nu=0}^{\infty} \frac{1}{(a s)^{\nu} \nu !} \prod_{x=1}^{n+s \nu}(a x+b)^{-1}, \quad n=0, \ldots, s .
$$

Тогда справедлива точная по высоте оценка

$$
\begin{gathered}
\left|h_{0} \psi_{0}+\ldots+h_{s} \psi_{s}\right| \gg H^{-s}(\ln (H+2) / \ln \ln (H+2))^{-(s+1) / 2}, \\
H=\max \left(\left|h_{0}\right|, \ldots,\left|h_{s}\right|\right)>0, \quad h_{0}, \ldots, h_{s} \in \mathbb{Z} .
\end{gathered}
$$

2. Многомерная цепная дробь Эйлера. Рассмотрим последовательность $(n=0,1, \ldots)$

$$
\psi_{n}=\sum_{\nu=0}^{\infty} \frac{1}{(s+1)^{\nu} \nu ! q^{n+(s+1) \nu}} \prod_{x=1}^{n+s \nu}((s+1)[x / s]+b(x))^{-1},
$$

где $s, q \in \mathbb{N}, b(k)=k(k=1, \ldots, s-1), b(s)=-1, b(x+s)=b(x)$. Покажем, что выполняется равенство

$$
\psi_{n-1}-((s+1)[n / s]+b(n)) q \psi_{n}=\psi_{n+s} .
$$

Действительно,

$$
\begin{aligned}
\psi_{n-1}- & ((s+1)[n / s]+b(n)) q \psi_{n} \\
= & \sum_{\nu=0}^{\infty} \frac{(s+1)[(n+s \nu) / s]+b(n)-(s+1)[n / s]-b(n)}{(s+1)^{\nu} \nu ! q^{n-1+(s+1) \nu}} \\
& \times \prod_{x=1}^{n+s \nu}((s+1)[x / s]+b(x))^{-1} \\
= & \sum_{\nu=1}^{\infty} \frac{1}{(s+1)^{\nu-1}(\nu-1) ! q^{n+s+(s+1)(\nu-1)}} \prod_{x=1}^{n+s \nu}((s+1)[x / s]+b(x))^{-1} \\
= & \psi_{n+s} .
\end{aligned}
$$

Следовательно, согласно лемме 2, справедливо разложение в правильную цепную $s$-дробь

$$
\begin{aligned}
& \left(\psi_{1} / \psi_{0}, \ldots, \psi_{s} / \psi_{0}\right)=\left[0 ; \vec{a}_{1}, \vec{a}_{2}, \ldots\right], \\
& \vec{a}_{n}=\left(0, \ldots, 0, a_{n}\right), \quad a_{n}=q((s+1)[n / s]+b(n)) .
\end{aligned}
$$


(Последовательность $a_{n}$ состоит из всех чисел вида $k q$, где $k \not \equiv 0(\bmod s+1), k \in \mathbb{N}$.)

Заметим, что при $k=0, \ldots, s,(34)$ преобразуется к виду

$$
\psi_{k}=\sum_{n=0}^{\infty} \frac{1}{((s+1) n+k) ! q^{(s+1) n+k}} .
$$

В частности, при $s=1$ получим

$$
\psi_{0}=\sum_{n=0}^{\infty} \frac{1}{(2 n) ! q^{2 n}}=\operatorname{ch}(1 / q), \quad \psi_{1}=\sum_{n=0}^{\infty} \frac{1}{(2 n+1) ! q^{2 n+1}}=\operatorname{sh}(1 / q)
$$

и из (35) получаем дробь Эйлера-Ламберта [8]

$$
\operatorname{th}(1 / q)=\left(e^{2 / q}-1\right) /\left(e^{2 / q}+1\right)=[0 ; q, 3 q, 5 q, \ldots] .
$$

Числа $\psi_{k}(k=0, \ldots, s)$ можно представить в виде линейной комбинации экспонент в алгебраических точках, например,

$$
\begin{array}{r}
\psi_{0}=\sum_{n=0}^{\infty} \frac{1}{((s+1) n) ! q^{(s+1) n}}=\frac{1}{(s+1)\left(e^{1 / q}+e^{p / q}+\ldots+e^{p^{s} / q}\right)}, \\
p=e^{2 \pi i /(s+1)} .
\end{array}
$$

При $s=3$ получим

$$
\begin{aligned}
& \psi_{0}=\sum_{n=0}^{\infty} \frac{1}{(4 n) ! q^{4 n}}=\frac{1}{2}(\operatorname{ch}(1 / q)+\cos (1 / q)), \\
& \psi_{1}=\sum_{n=0}^{\infty} \frac{1}{(4 n+1) ! q^{4 n+1}}=\frac{1}{2}(\operatorname{sh}(1 / q)+\sin (1 / q)), \\
& \psi_{2}=\sum_{n=0}^{\infty} \frac{1}{(4 n+2) ! q^{4 n+2}}=\frac{1}{2}(\operatorname{ch}(1 / q)-\cos (1 / q)), \\
& \psi_{3}=\sum_{n=0}^{\infty} \frac{1}{(4 n+3) ! q^{4 n+3}}=\frac{1}{2}(\operatorname{sh}(1 / q)-\sin (1 / q))
\end{aligned}
$$

и (35) запишется в виде трехмерной цепной дроби

(36) $\quad\left(\psi_{1} / \psi_{0}, \psi_{2} / \psi_{0}, \psi_{3} / \psi_{0}\right)$

$$
=\frac{1}{(0,0, q)+\frac{1}{(0,0,2 q)+\frac{1}{(0,0,3 q)+\frac{1}{(0,0,5 q)+\ldots}}}} .
$$

Оценим величины $H_{n}=a_{n-s} a_{n-2 s} \ldots a_{r}(1 \leq r \leq s)$. 
Лемма 6. Справедливы оценки

$$
\begin{gathered}
\ln H_{n} \asymp n \ln n, \\
H_{n+1} / H_{n} \asymp n^{[(n+1) / s]-[n / s]+(b(n+1)-b(n)) /(s+1)}, \\
H_{n+1} \ldots H_{n+s} \asymp H_{n}^{s} n^{\beta(n)},
\end{gathered}
$$

где $\beta(n)=1+n-s[n / s]+(b(1)+\ldots+b(s)-s b(n)) /(s+1)$.

Доказательство.Оценка (37) получается аналогично (32). Проверим (38):

$$
\begin{aligned}
\frac{H_{n+1}}{H_{n}} \asymp & \prod_{k=1}^{[n / s]} \frac{(s+1)[(n+1-k s) / s]+b(n+1)}{(s+1)[(n-k s) / s]+b(n)} \\
& =\prod_{k=1}^{[n / s]}\left(1+\frac{[(n+1) / s]-[n / s]+(b(n+1)-b(n)) /(s+1)}{([n / s]-k+b(n)) /(s+1)}\right) .
\end{aligned}
$$

Так же как при выводе (33) получим

$\ln \left(H_{n+1} / H_{n}\right)=O(1)+([(n+1) / s]-[n / s]+(b(n+1)-b(n)) /(s+1)) \ln n$, откуда следует (38).

Оценка (39) является следствием (38). Действительно,

$$
\begin{aligned}
& H_{n+1} \asymp n^{[(n+1) / s]-[n / s]+(b(n+1)-b(n)) /(s+1)} H_{n}, \\
& H_{n+2} \asymp n^{[(n+2) / s]-[n / s]+(b(n+2)-b(n)) /(s+1)} H_{n}, \ldots, \\
& H_{n+s} \asymp n^{[(n+s) / s]-[n / s]+(b(n+s)-b(n)) /(s+1)} H_{n} .
\end{aligned}
$$

Перемножая эти оценки, получим (39):

$$
H_{n+1} \ldots H_{n+s} \asymp H_{n}^{s} n^{\beta(n)},
$$

где

$$
\begin{aligned}
\beta(n)= & {[(n+1) / s]+\ldots+[(n+s) / s]-s[n / s] } \\
& +\frac{b(n+1)+\ldots+b(n+s)-s b(n)}{s+1} \\
= & 1+n-s[n / s]+\frac{b(1)+\ldots+b(s)-s b(n)}{s+1} .
\end{aligned}
$$

Лемма доказана.

Заметим, что $\beta(n+s)=\beta(n)$ и

$$
\max _{k=1, \ldots, s} \beta(k)=\beta(s)=1+\frac{2+\ldots+s}{s+1}=\frac{s(s+3)}{2(s+1)} .
$$

В силу леммы 6 условия (24) выполнены и

$$
H_{n+1} \ldots H_{n+s} \ll H_{n}^{s}\left(\ln \left(H_{n}+2\right) / \ln \ln \left(H_{n}+2\right)\right)^{s(s+3) /(2 s+2)},
$$


причем для $n \equiv 0(\bmod s)$,

$$
H_{n+1} \ldots H_{n+s} \asymp H_{n}^{s}\left(\ln \left(H_{n}+2\right) / \ln \ln \left(H_{n}+2\right)\right)^{s(s+3) /(2 s+2)} .
$$

Применяя теорему 2 , получаем следующее утверждение:

Teopema 3. Пусть $s, q \in \mathbb{N} u$

$$
\psi_{k}=\sum_{n=0}^{\infty} \frac{1}{((s+1) n+k) ! q^{(s+1) n+k}}, \quad k=0, \ldots, s .
$$

Тогда справедлива точная по высоте оченка

$$
\begin{gathered}
\left|h_{0} \psi_{0}+\ldots+h_{s} \psi_{s}\right| \gg H^{-s}(\ln (H+2) / \ln \ln (H+2))^{-s(s+3) /(2 s+2)}, \\
H=\max \left(\left|h_{0}\right|, \ldots,\left|h_{s}\right|\right)>0, \quad h_{i} \in \mathbb{Z} .
\end{gathered}
$$

В частности, при $s=3$, выполняется точная по высоте оченка

$$
\begin{gathered}
\left|h_{0} \operatorname{ch}(1 / q)+h_{1} \cos (1 / q)+h_{2} \operatorname{sh}(1 / q)+h_{3} \sin (1 / q)\right| \\
\gg H^{-3}(\ln (H+2) / \ln \ln (H+2))^{-9 / 4}, \\
H=\max \left(\left|h_{0}\right|,\left|h_{1}\right|,\left|h_{2}\right|,\left|h_{3}\right|\right)>0, \quad h_{0}, h_{1}, h_{2}, h_{3} \in \mathbb{Z} .
\end{gathered}
$$

3. Многомерная иепная дробь Рамануджана. Пусть $a, s \in \mathbb{N}, a \geq 2$. Рассмотрим последовательность

$$
\begin{array}{r}
\psi_{n}=a^{-n(n+1) / 2} \sum_{\nu=0}^{\infty} a^{(-s(s+1) / 2) \nu^{2}-(s+1) n \nu} \prod_{k=1}^{\nu}\left(a^{(s+1) k}-1\right)^{-1}, \\
n=0,1, \ldots
\end{array}
$$

Покажем, что справедливо равенство

$$
\psi_{n-1}-a^{n} \psi_{n}=\psi_{n+s}, \quad n=1,2, \ldots
$$

Действительно,

$$
\begin{aligned}
\psi_{n-1}= & a^{n} \psi_{n} \\
= & a^{-n(n-1) / 2} \\
& \times \sum_{\nu=0}^{\infty} a^{(-s(s+1) / 2) \nu^{2}}\left(a^{-(s+1)(n-1) \nu}-a^{-(s+1) n \nu}\right) \prod_{k=1}^{\nu}\left(a^{(s+1) k}-1\right)^{-1} \\
= & a^{-n(n-1) / 2} \sum_{\nu=1}^{\infty} a^{(-s(s+1) / 2) \nu^{2}-(s+1) n \nu} \prod_{k=1}^{\nu-1}\left(a^{(s+1) k}-1\right)^{-1} \\
= & a^{-n(n-1) / 2-n(s+1)-s(s+1) / 2} \\
& \times \sum_{\nu=0}^{\infty} a^{(-s(s+1) / 2) \nu^{2}-(s+1)(n+s) \nu} \prod_{k=1}^{\nu}\left(a^{(s+1) k}-1\right)^{-1} \\
= & \psi_{n+s} .
\end{aligned}
$$


В силу леммы 2 , справедливо разложение в правильную $s$-дробь

$$
\left(\psi_{1} / \psi_{0}, \ldots, \psi_{s} / \psi_{0}\right)=\frac{1}{(0, \ldots, a)+\frac{1}{\left(0, \ldots, a^{2}\right)+\ldots}} .
$$

При $s=1$ из (40) и (41) получаем цепную дробь Рамануджана [14] (см. также [12] и [6])

$$
\begin{aligned}
\psi_{0} & =\sum_{n=0}^{\infty} a^{-n^{2}} \prod_{k=1}^{n}\left(a^{2 k}-1\right)^{-1}, \\
\psi_{1} & =\sum_{n=0}^{\infty} a^{-(n+1)^{2}} \prod_{k=1}^{n}\left(a^{2 k}-1\right)^{-1}, \\
\psi_{1} / \psi_{0} & =\left[0 ; a, a^{2}, a^{3}, \ldots\right], \quad a \in \mathbb{N}, a \geq 2 .
\end{aligned}
$$

Пусть

$$
H_{n}=a^{n-s} a^{n-2 s} \ldots a^{r} \quad(1 \leq r \leq s) .
$$

Тогда

$$
\begin{gathered}
\log _{a} H_{n}=\sum_{k=1}^{[n / s]}(n-k s)+O(1)=n^{2} /(2 s)+O(n), \\
n=\sqrt{2 s}\left(\log _{a} H_{n}\right)^{1 / 2}+O(1), \\
H_{n+1} / H_{n} \asymp a^{n / s}, \quad H_{n+k} \asymp a^{k n / s} H_{n} \quad(k=1, \ldots, s), \\
H_{n+1} \ldots H_{n+s} \asymp a^{n(s+1) / 2} H_{n}^{s} \asymp H_{n}^{s+(s+1) \sqrt{s / 2} / \sqrt{\log _{a} H_{n}}} .
\end{gathered}
$$

Условия (24), очевидно, выполнены и, применяя теорему 2, получаем точную по высоте оценку

$$
\begin{gathered}
\left|h_{0} \psi_{0}+\ldots+h_{s} \psi_{s}\right| \gg H^{-s-(s+1) \sqrt{s} / \sqrt{2 \log _{a} H}}, \\
H=\max \left(\left|h_{0}\right|, \ldots,\left|h_{s}\right|\right)>0, \quad h_{0}, \ldots, h_{s} \in \mathbb{Z}, a \geq 2, a \in \mathbb{N},
\end{gathered}
$$

а $\psi_{0}, \ldots, \psi_{s}$ определены равенством (40).

З ам еч ан и я. 1. Впервые неулучшаемые оценки линейных форм были получены в работе автора [3] в 1981 г. Затем обобщение этого результата опубликовал А. И. Галочкин [1] в 1984 г. (в 1983 г. им было опубликовано краткое сообщение на эту тему). Новыми результатами статьи являются многомерные обобщения цепных дробей Эйлера, Гурвица и Рамануджана и соответствующие точные оценки линейных форм. Теорема 2 повторяет результат автора [3] для иллюстрации нового подхода. Очевидно, общая теорема 1 позволяет строить множество других примеров чисел, заданных явно цепной дробью, для которых будут получаться новые неулучшаемые оценки линейных форм. 
2. Теорему 3 не следует рассматривать как известный пример на применение теоремы из [1], поскольку:

а) Главный смысл теоремы 3 состоит в иллюстрации естественности нового обобщения многомерной дроби Эйлера (36), в которой разлагаются линейные комбинации экспонент в алгебраических точках.

б) Возможность получения теоремы 3 как следствия теоремы из [1] становится понятной в связи с доказательством теоремы 3. Вид функций в теореме 3 отличается от рассматривавшихся в работах [3] и [1] тем, что в них не присутствует явно множитель $1 / \nu$ !.

в) Теорема 3 легко может быть выведена методом работы автора [3], если последовательность $a x+b$ заменить на $a x+b(x)$, где $b(x+s)=$ $b(x)$, и выбрать затем $a=q(s+1) / s, b=q\{(x-1) / s\}-q / s, z=1$ $(s, q, x, a x+b(x) \in \mathbb{N})$.

г) Ни в одной из известных автору работ не рассматривались примеры с точными оценками линейных форм для функций из теоремы 3 (за исключением тривиального случая $s=1$ ).

3. В некоторых утверждениях второго параграфа, в которых это явно оговорено, предполагается линейная независимость компонент вектора вместе с 1 над $\mathbb{Q}$. В леммах $1-5$ и теоремах $1-3$ такая линейная независимость не предполагается в формулировках и не используется в доказательствах. Линейная независимость следует из теоремы 1 , и соответствующая цепная дробь может быть получена по алгоритму, описанному во втором параграфе.

4. Статья представляет собой изложение результатов 3-й главы кандидатской диссертации автора Цепные дроби и диофантовы приближения, Москва 1990 (на правах рукописи), выполненной в 198488 г.г. в МГУ им. М. В. Ломоносова.

\section{Литература}

[1] А. И. Галочкин, О неулучшаемых по высоте оченках некоторых линейных форм, Мат. сб. 124 (1984), 416-430.

[2] -, Уточнение оценок некоторых линейных форм, Мат. заметки 20 (1976), $35-45$.

[3] А. Н. Коробов, Оценки некоторых линейных форм, Вест. МГУ сер. мат.мех. 6 (1981), 36-40.

[4] С. Ленг, Введение в теорию диофантовых приближений, Мир, Москва, 1970.

[5] Е. М. Никишин, В. Н. Сорокин, Рациональнье аппроксимации и ортогональность, Наука, Москва, 1988. 
[6] Б. Г. Тасоев, Некоторые вопросы теории непрерывных дробей, Труды Тбилисс. унив. мат. мех. астроном. 16-17 (1984), 53-83.

[7] А. Я. Хинчин, Цепнье дроби, Наука, Москва, 1978.

[8] Л. Эйлер, Введение в анализ бесконечно мальх, ОНТИ, Москва, 1936.

[9] W. Adams, Asymptotic Diophantine approximations and Hurwitz numbers, Amer. J. Math. 83 (1967), 1083-1108.

[10] L. Bernstein, The Jacobi-Perron Algorithm. Its Theory and Application, Lecture Notes in Math. 207, Springer, 1971.

[11] A. J. Brentjes, Multi-Dimensional Continued Fraction Algorithms, Math. Centrum, Amsterdam, 1981.

[12] G. H. Hardy and E. M. Wright, Introduction to the Theory of Numbers, 4th ed., Oxford Univ. Press, London, 1960.

[13] O. Perron, Grundlagen fur eine Theorie des Jacobischen Kettenbruch Algorithmus, Math. Ann. 64 (1907), 1-76.

[14] S. Ramanujan, Collected Papers, Cambridge Univ. Press, 1927.

РОССИЙСКАЯ КНИЖНАЯ ПАЛАТА

ПРОЕЗД РУСАНОВА Д. 23 КВ. 87

МОСКВА 129323, РОССИЯ

Поступило 12.2.1992

и в дополненной форме 7.5.1994 